\title{
Binding of VEGF-A to canine cancer cells with preferential expression of VEGFR1
}

\author{
Antonella Borgatti ${ }^{1,2}$, Megan Duckett ${ }^{1,2}$, Charles Spangler ${ }^{3}$ and Jaime F. Modiano ${ }^{1,2}$
}

1. Department of Veterinary Clinical Sciences, College of Veterinary Medicine, University of Minnesota, 1352

Boyd Avenue, St Paul, MN 55108, USA; 2. Masonic Cancer Center, University of Minnesota, 420 Delaware Street SE, Minneapolis, MN 55455, USA; 3. SensoPath Technologies, 920 Technology Blvd, Bozeman, MT 59718, USA

Corresponding author: Antonella Borgatti, email: borgatti@umn.edu, Tel: 612-626-5786

Received: 12-10-2013, Revised: 21-11-2013, Accepted: 26-11-2013, Published online: 02-01-2014

doi: $10.14202 /$ vetworld.2014.1-6

How to cite this article: Borgatti A, Duckett M, Spangler C, Modiano JF (2014) Binding of VEGF-A to canine cancer cells with preferential expression of VEGFR1, Veterinary World 7(1): 1-6.

\begin{abstract}
Aim: Despite encouraging results in syngeneic and xenografts cancer models with various inhibitors of vascular endothelial growth factor (VEGF) or its receptors (VEGFRs), beneficial effects have not been consistently translated to the clinic, underscoring the need to develop strategies that go beyond the inhibition of these targets. The purpose of this study was to generate data to support the hypothesis that VEGF may be used as "bait" to selectively deliver therapeutics to VEGFRexpressing cancer cells.

Materials and Methods: VEGFR1 and VEGFR2 expression was characterized using real time quantitative reverse transcriptase polymerase chain reaction (RT-qPCR) in canine hemangiosarcoma (Grace-HSA, Emma-HSA), melanoma (TLM-1), and thyroid adenocarcinoma (CTAC) cell lines. TLM-1 and Grace-HSA were identified as representative cell lines that selectively expressed high levels of VEGFR1. Flow cytometry was performed to examine binding of a single VEGF molecule (biotinylated VEGFA and avidin conjugated to fluorescein isothiocyanate (FITC)) by these chemoresistant cell lines.

Results: RT-qPCR showed that canine tumor cells can preferentially express VEGFR1 over VEGFR2. Both TLM-1 and Grace-HSA cell lines, which represent VEGFR1-expressing tumors, showed specific binding to VEGF-A and this binding was competitively inhibited by anti-VEGF antibody.

Conclusions: Cells preferentially expressing VEGFR1 can be targeted with a single VEGF molecule and these ligandreceptor pairs are well suited for targeting cytotoxic molecules in various canine tumor cells. Further studies are needed to develop strategies to selectively deliver therapeutics through VEGF-VEGFRs binding into VEGFR-expressing tumors.
\end{abstract}

Key words: canine, dog, delivery, target, vascular endothelial growth factor, vascular endothelial growth factor receptor

\section{I ntroduction}

Vascular endothelial growth factors (VEGF) are key regulators of angiogenesis and of lymphatic and blood vessel function in both health and disease condition in the adult [1]. VEGF-A (the prototype) is widely expressed by nearly all human malignant tumors [1], as well as by various canine tumors, such as mammary carcinoma [2], fibrosarcoma, osteosarcoma, melanoma, carcinoma [3], and hemangiosarcoma (HSA) $[2,4,5]$. Physiologically, VEGF stimulates the formation of new blood vessels and regulates their structure and function. This regulatory effect is lost in VEGF-induced tumor blood vessels, which are disordered, tortuous, and leaky, resulting in high tumor interstitial pressure. The increase in vascular permeability that results from increased VEGF signaling leads to poor perfusion and hypoxia, which in turn, further stimulates VEGF production. VEGF is believed to act through three receptor tyrosine kinases (RTK), VEGFR1 (Flt1), VEGFR2 (Flk1), and VEGFR3

Copyright: The authors. This article is an open access article licensed under the terms of the Creative Commons Attribution License (http://creativecommons.org/licenses/by/2.0) which permits unrestricted use, distribution and reproduction in any medium, provided the work is properly cited.
(Flt4). VEGFR1 is primarily expressed in monocytes and macrophages, VEGFR2 in vascular endothelial cells and their precursors, and VEGFR3 in lymphatic endothelial cells, vascular endothelial cells, and nonendothelial compartments such as neuronal progenitors, osteoblasts, and macrophages [6]. VEGFR3 is overexpressed during angiogenesis, but its expression in cancer cells remains a matter of debate [6]. VEGFR2 is the canonical endothelial cell receptor that transmits growth and survival signals and is considered the main transducer of VEGFA effects on endothelial cell differentiation, proliferation, migration, and neovascularization during physiologic and pathologic conditions [6]. Conversely, the function and biology of VEGFR1 is more elusive. The physiological ligand of VEGFR1 is placental-derived growth factor (PGF). VEGFR1 has been described as a possible negative regulator of VEGFR2 biology via binding and sequestering of VEGF; it has also been reported to regulate monocyte migration during inflammation [6]. A variety of pathologic conditions, including tumor growth (glioma), metastasis, and inflammatory diseases (e.g. rheumatoid arthritis) are sensitive to loss or inhibition of VEGFR1 function $[7,8]$. Possible positive and negative roles for VEGFR1 
in the transmission of growth and survival signals also have been suggested [6].

Various drugs targeting VEGF-A or VEGFRs, including antibodies, bioengineered molecules that mimic VEGFRs, and small molecule RTK inhibitors, can prevent tumor growth in syngeneic and xenograft mouse models [1]. Nevertheless, these beneficial effects have not been consistently replicated in the clinic. For example, despite success in certain, such as metastatic colorectal cancer [9], adjuvant bevacizumab (Avastin; Genentech) did not provide a survival advantage to patients with metastatic breast cancer compared to those treated with chemotherapy [10]. In 2011, the Food and Drug Administration (FDA) revoked the approval of bevacizumab for breast cancer patients due to potentially life-threatening side effects without proof of survival or quality of life benefits. Other FDA-approved VEGFA/VEGFR inhibitors have similarly yielded disappointing results [11]. Furthermore, VEGFR inhibitors suppressed early tumor growth in transgenic models of islet cell adenocarcinoma, but this was not achieved in advanced tumors, presumably due to increased pericyte coverage in the maturing vessels [1]. Notably, the lack of benefit of these strategies in the clinic goes in parallel with their possible detrimental consequences. In fact, cancer cells that are not killed may be rendered hypoxic, leading to further expression of VEGFA and other pro-angiogenic growth factors [1].

Noteworthy, there are striking structural similarities shared amongst canine and human VEGF and VEGFRs. The canine and human sequences for VEGF, VEGFR1 and VEGFR2 are 95\%, 90\%, and 93\% identical at the amino acid level, and all retain $95-96 \%$ homology when conserved substitutions are considered. These similarities are based on published sequences available through the National Center for Biotechnology Information, National Library of Medicine and Ensemble. Gene IDs (NCBI) are as follows: canine VEGFA: 403802, human VEGFA: 7422; canine VEGFR1: 403727, human VEGFR1: 2321; canine VEGFR2: 482154, human VEGFR2: 3791. Their almost identical biological and cell-binding properties suggest that the identification of canine tumors expressing VEGF and VEGFR and the optimization of targeted anti-cancer strategies in dogs will provide important insights in the treatment of humans with similar diseases. Because drugs that inhibit VEGF/ VEGFR pathways might not improve patient outcomes beyond what is achievable by standard of care therapy, novel approaches that go beyond the inhibition of these targets are necessary.

A promising strategy is to use VEGF as a binding molecule ("bait") to selectively deliver therapeutic agents, such as de-immunized toxins, radionucleides, photodynamic therapy compounds, and chemotherapeutics, to VEGFR-expressing cancer cells and neoangiogenic endothelium [12,13]. Work by Hamada and colleagues using a VEGF-conjugated fluorescence nanoparticle showed staining of VEGFR-expressing cells during angiogenesis in an ischemic mouse model, confirming successful binding by the VEGF moiety to VEGFR2 [14]. While VEGFR1 and VEGFR2 expression has been confirmed in various human tumors and neoangiogenic vascular cells, little is known about their expression in canine tumors. Our group previously showed a heretofore-unrecognized pattern of VEGFR expression in canine HSA that appears to be modulated by dogs' genetic background [4]. Cultured HSA cells were resistant to VEGFR2 inhibition, with no change in magnitude or kinetics of cell growth. In contrast, when VEGFR1 was inhibited, tumors that overexpressed VEGFR1 showed greater proliferation, supporting the role of VEGFR1 as an active growth inhibitor. This suggests VEGFR1-expressing cells should be targetable with VEGF-conjugates. Thus, in this study, we preliminarily investigated the feasibility of our proposed strategy that VEGF may be used as "bait" to deliver therapeutics to VEGFR1-expressing cancer cells. The hypothesis of our study was that canine cells that preferentially express VEGFR1 could be efficiently bound by VEGF-A. To the authors' knowledge, this is the first time that binding of VEGFA to VEGFR1 has been tested in canine cancer cells and similar studies have not been previously conducted in humans. While evaluating the efficacy of this possible therapeutic strategy is beyond the scope of this hypothesis-generating study, the effective binding of VEGF-A to VEGFR-1 suggests that if therapeutics could be selectively delivered to VEGFR-1 expressing cancer cells, this may result in greater anticancer activity than what has been achieved with drugs inhibiting VEGF receptors. Importantly, given the aforementioned similarities shared amongst canine and human VEGF and VEGFRs, the successful application of this strategy in dogs may establish the foundation to extend this approach to human cancer patients.

\section{Materials and Methods}

Cell cultures: The canine hemangiosarcoma cell lines Grace-HSA and Emma-HSA, the melanoma cell line TLM-1, and the thyroid adenocarcinoma cell line CTAC were maintained as adherent cultures at $37^{\circ} \mathrm{C}$ in $5 \% \mathrm{CO} 2$ atmosphere as described [4,15-17].

Real time quantitative reverse transcriptase polymerase chain reaction ( $R T$ - PPCR): Elimination of genomic DNA and reverse transcription were both carried out using QuantiTect Reverse Transcription Kit (Qiagen, Valencia, CA). RT-qPCR was done as described using an Eppendorf Mastercycler ep realplex with FastStart SYBR Green Master Mix Protocol (Roche, Indianapolis, IN) [18]. Primer sequences are provided in Table-1. GAPDH was used as the reference standard for normalization and relative levels of steady state mRNA were established using the comparative [delta][delta]Ct method (Livak) 2001 [19]. 
Table 1. Primer Sequences for RT-qPCR

\begin{tabular}{lllc}
\hline Gene & Forward primer (sense, 5'-3') & Reverse primer (antisense, 5'-3') & Product size \\
\hline VEGFR1 & ATCTGCCTGTGGAAGGAATG & CGGGTATTTCACTGTGCATC & 200 \\
VEGFR2 & CTATGTGTGCTTCGCTCAGG & GTGAGCTGCTGAGTCTTCCA & 213 \\
GAPDH & GGAGTCCACTGGCGTCTTCAC & GAGGCATTGCTGATGATCTTGAGG & 165 \\
\hline
\end{tabular}

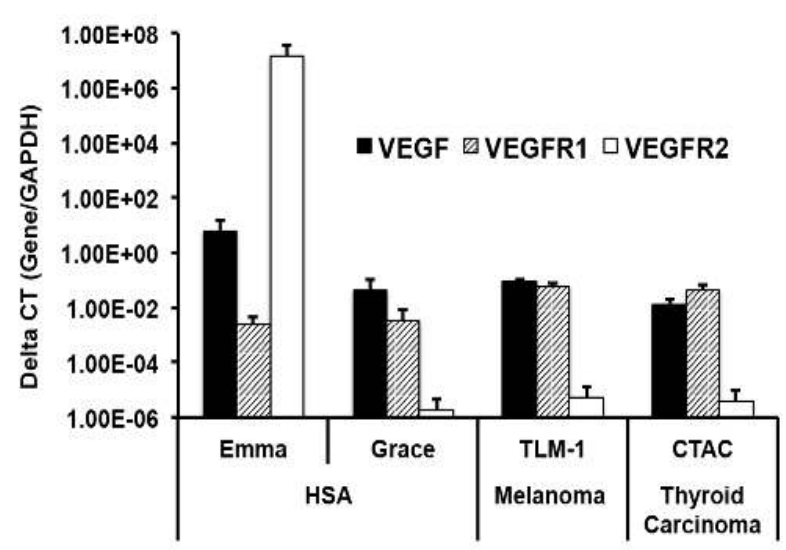

Figure-1. VEGFR expression was present in all canine cancer cell lines tested. RT-qPCR was performed using an Eppendorf Mastercycler ep realplex with FastStart SYBR Green Master Mix Protocol. Expression of VEGF, VEGFR1, and VEGFR2 was evaluated in Emma-HSA, Grace-HSA, TLM-1, and CTAC cell lines. Relative expression for each of these genes is shown normalized to GAPDH.

Cytotoxicity assays: Cells were incubated in 96 well plates overnight prior to addition of paclitaxel at the concentrations indicated in the results section. Conditions were performed in triplicate using 5,000 cells per well in $100 \mu \mathrm{l}$ of culture medium. Cytotoxic responses were assessed using a non-radioactive, colorimetric cell proliferation (MTD) assay. Viability was assessed after $72 \mathrm{hr}$ of culture using CellTiter96 AQueous kit (Promega, Madison, WI) as described by the manufacturer.

Flow cytometry: Cells were harvested using Accutase and resuspended in staining buffer (PBS with 2\% FBS). VEGF binding was measured using the human VEGF biotinylated fluorokine kit (R\&D Systems, Minneapolis, MN) as described by the manufacturer. Cells were analyzed using a FACSCalibur cytometer (BD Biosciences, San Jose, CA) and data were analyzed with FlowJo (v9.5.2) software (Treestar, Ashland, OR). Viable cells were determined by exclusion of 7-Aminoactinomycin D (7AAD).

\section{Results}

TLM-1 and Grace-HSA cells show preferential expression of VEGFR1: Expression of VEGF, VEGFR1, and VEGFR2 has been confirmed in a variety of human tumors and neoangiogenic vascular cells, but little is known about their expression in canine tumors. A pattern of VEGFR expression modulated by dogs' genetic background was previously shown in canine HSA cells [4]. Here, we used RT-qPCR to characterize expression of VEGF, VEGFR1 and VEGFR2 genes in four different canine cancer cells lines to identify tumors that preferentially expressed VEGFR1.

Figure-1 illustrates expression of VEGF and

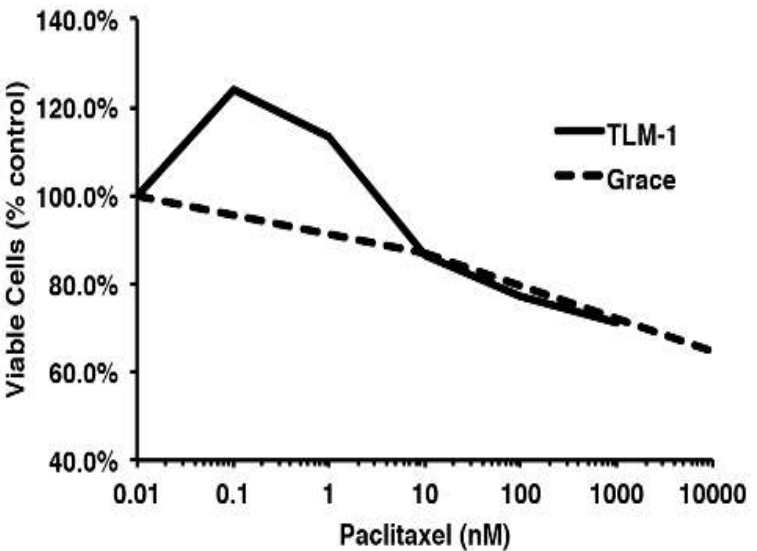

Figure-2. TLM- 1 and Grace-HSA cell lines are resistant to paclitaxel (Taxol). Canine HSA (Grace-HSA), dashed line, and melanoma (TLM1 ), solid line, cell lines were cultured for $72 \mathrm{hr}$ with increasing concentrations of paclitaxel (Taxol). Viability was measured in triplicate samples using the Cell Titer $96 \AA$ Aqueous Non-Radioactive Cell Proliferation Assay (MTS)

VEGF receptors by two HSA cell lines (Emma-HSA and Grace-HSA), one melanoma cell line (TLM-1), and one thyroid adenocarcinoma cell line (CTAC). Expression of VEGF was remarkably consistent, and VEGFR expression was confirmed in all cell lines tested. TLM-1 and Grace-HSA cells were identified as representative cell lines that retained preferential expression of VEGFR1 with significantly reduced expression of VEGFR2. These cell lines were selected to verify their chemoresistance and to test VEGF binding in subsequent experiments as described below.

TLM-1 and Grace-HSA cell lines are resistant to paclitaxel (Taxol): Our group has previously shown that TLM-1 cells are highly resistant to various conventional chemotherapy agents. In fact, the halfmaximal inhibitory concentrations $\left(\mathrm{IC}_{50}\right)$ for TLM-1 growth inhibition with doxorubicin, camptothecin, and 5-fluorouracil were all $>1 \mu \mathrm{M}$ in standard $72 \mathrm{hr}$ cytotoxicity assays (R. Weiss and J. Modiano, unpublished data). Similarly, HSA cells are highly resistant to conventional chemotherapeutics [20]. Chemoresistance is one of the greatest limitations in the successful treatment of many solid tumors. It is unclear if it is due to the use of conservative chemotherapy regimen, or to properties that are intrinsic to the specific cancer cells. To further examine the sensitivity of TLM-1 and Grace-HSA cells to chemotherapy, we determined in vitro cytotoxicity of paclitaxel, a potent anti-mitotic, against these two cell lines. As shown in Figure-2, only minimal cytotoxicity was seen for both TLM1 and Grace-HSA cells following exposure to increasing concentrations of paclitaxel $\left(\mathrm{IC}_{50}>10 \mu \mathrm{M}\right)$. This corroborates the clinical experience that HSA and melanoma cells are strongly chemoresistant. 


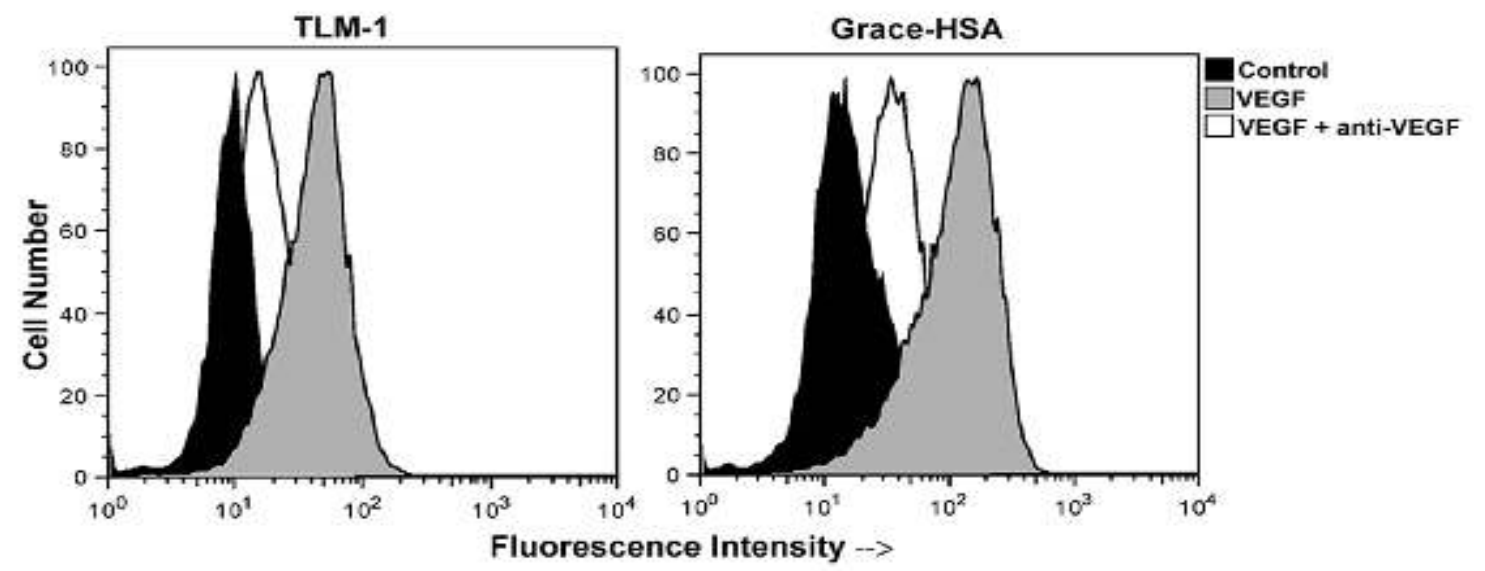

Figure-3. VEGF-A binds to both VEGFR1 and VEGFR2. One dimensional histograms illustrating fluorescence ( $x$-axis) relative to cell number (y-axis) for both cell lines. The black peak represents the negative control. The grey peak represents human recombinant VEGF conjugated to fluorescein isothiocyanate (FITC). The white peak represents VEGF with blocking from anti-VEGF antibodies, to illustrate specificity. VEGF binding was measured using the human VEGF biotinylated fluorokine kit (R\&D Systems, Minneapolis, MN) and cells were analyzed using a FACSCalibur cytometer (BD Biosciences, San Jose, CA).

VEGF-A binds to cells expressing VEGFR1: In contrast to the limited efficacy of several chemotherapy regimens against many solid malignancies, HSA cells are sensitive to cytotoxic compounds such as genetically engineered bacterial toxins [20]. If these compounds could be conjugated to ligands such as VEGF, this may allow selective targeting of VEGFRexpressing cancer cells. Thus, to further investigate the feasibility of targeting such compounds to VEGFR1expressing tumors, we used flow cytometry to examine VEGF binding in TLM-1 and Grace-HSA cells. Figure- 3 illustrates that both TLM-1 and Grace-HSA cell lines showed specific binding to VEGF-A. Binding was inhibited by competition with the anti-VEGF antibody, but intriguingly, there was significantly greater receptor density ( 1-log) in Grace-HSA cells than in TLM-1 cells as determined both by the mean fluorescence intensity at the same concentration of ligand, and by the blunted inhibition at the same concentration of antibody. This suggests that despite having lower steady state levels of VEGFR mRNA (in relation to GAPDH) than melanoma cells, HSA cells might use post-translational mechanisms to stabilize expression of VEGFRs on the cell surface.

Indeed, the binding of biotinylated VEGF to Grace-HSA cells was significantly reduced when unlabeled VEGF was used as a competitor at 100-fold molar excess $(500 \mathrm{ng} / \mathrm{ml})$ but not at 10 -fold excess molar excess. We believe this is due to the relatively slow rate of ligand binding at $4 \mathrm{C}$, so that even after 15 min pre-incubation, unlabeled VEGF used at $50 \mathrm{ng} / \mathrm{ml}$ did not occupy all available receptor molecules to saturation. Endogenous VEGF produced by the cells did not affect the assay, indicating the tumor cells were capable of binding exogenous VEGF and were thus amenable to targeting.

\section{Discussion}

Our cytotoxicity experiments showed that both TLM-1 cells and Grace-HSA cells were resistant to paclitaxel at micromolar concentrations, corroborating the clinical experience that HSA and melanoma cells are strongly chemoresistant. Despite low levels of VEGFR2 expression, these cells could still be targeted by VEGF, suggesting that tumors that preferentially express VEGFR1 can be viably targeted using VEGFconjugates. This effect could be additive with targeting of stromal endothelial cells that express high levels of VEGFR2. Interestingly, since VEGF-targeted compounds are not substrates for chemoresistance mechanisms, they may facilitate penetration of chemotherapeutics into tumor and neoangiogenic cells, which may result in enhanced anti-tumor activity by means of selective targeting rather than VEGFR inhibition. It has to be acknowledged that normal cells expressing VEGFRs outside the tumor environment might be killed along with VEGFR-expressing cancer cells, leading to adverse effects of the treatment, including vascular leak syndrome (VLS), hemolytic uremic syndrome, liver, renal, or cardiac damage. Furthermore, binding of targeted drugs might enhance the overall function of VEGFRs. Conversely, it cannot be completely excluded that binding with VEGF-A might neutralize the effect of those cytotoxic drugs. However, the latter seems unlikely given that previous studies using VEGFR-targeting compounds as well as bioengineered ligand-targeted agents showed potent antitumor activity both in vitro and in vivo in experimental mouse models [12, 13] with no loss of anti-proliferative potential due to binding by a specific targeting molecule. These studies also suggested that the structural changes occurring in the ligands following conjugation with a binding molecule did not interfere with the ability of the ligands to bind their respective receptors. For example, the bispecific ligand targeted toxin EGFuPA made up of the epidermal growth factor (EGF) and the urokinase plasminogen activator (UPA) conjugated to a truncated Pseudomonas exotoxin (PE) A (PE38) was previously shown to have potent anticancer activity via inhibition of protein synthesis [21]. Pre-clinical work in vitro and in mice, respectively, showed highly efficient targeting and killing of canine hemangio- 
sarcoma (HSA) cells and HSA tumor initiating cells at clinically achievable concentrations of EGFuPA, and that EGFuPA targets chemotherapy-resistant tumor cells and the associated tumor microvasculature [20-22]. Novel VEGFR-targeting compounds designed to deliver chemotherapeutics as well as other anticancer agents to VEGFR-expressing cancer cells may exhibit similar anticancer activity. Preclinical studies confirming successful targeting and investigating the potential toxicity of novel compounds delivering therapeutics to VEGFR-expressing cells are essential prior to clinical translation of the proposed strategy.

Based on our RT-qPCR data, we selected TLM-1 and Grace-HSA cells as representative lines with enrichment of VEGFR1 expression to test VEGF binding. Our results confirmed a successful VEGFbinding that did not appear to be affected by endogenous VEGF. The greater fluorescence noted in Grace-HSA compared to TLM-1 might be due to lineage-specific post-translational stabilization leading to increased receptor density. Not surprisingly, competition with unlabeled VEGF was more efficient in TLM1 cells that appeared to have decreased surface receptors density compared to Grace-HSA. Considering systemic concentrations of VEGF in tumor bearing dogs are in the picomolar range $[23,24]$, receptors in tumor cells should be readily available for targeting under most conditions, as plasma levels of VEGF have been shown to be $<100 \mathrm{pg} / \mathrm{ml}$ for $95-100 \%$ of tumor-bearing dogs [23,24].

\section{Conclusions}

In summary, canine tumor cells can show preferential expression of VEGFR1, but these cells remain capable of binding native VEGF-A, thus providing feasibility of targeting VEGFR-expressing tumor cells with cytotoxic molecules conjugated to VEGF-A. Optimization of anti-cancer strategies targeting VEGFR1 receptors in dogs as part of the process of clinical translation could provide insights on their potential efficacy and toxicity [25]. Further studies are needed to develop strategies based on the selective delivery of therapeutic compounds against VEGFR-expressing tumors through VEGF-VEGFRs binding.

\section{Authors' contributions}

$\mathrm{AB}, \mathrm{CS}$, and JFM conceptualized the study. AB and JFM obtained funding. AB and MD performed and analyzed the experiments, and wrote the initial draft of the manuscript. AB, MD, CS, JFM reviewed and edited all subsequent drafts of the manuscript. All authors read and approved the final manuscript.

\section{Acknowledgements}

We thank the American College of Veterinary Internal Medicine (ACVIM) Foundation and the Canine Health Foundation and the Animal Cancer Care and Research Program of the University of Minnesota for providing the funding that allowed the conduction of our experiments, and Dr. Brenda Spangler for helpful discussions.

\section{Competing interests}

The authors declare that they have no competing interests.

\section{References}

1. Sitohi, B., Nagy, J.A. and Dvorak, H.F. (2012) Anti-VEGF / VEGFR therapy for cancer: reassessing the target. Cancer Res., 72(8): 15, 1909-1914

2. Restucci, B., Paparella, S., Maiolino, P. and De Vico, G. (2002) Expression of vascular endothelial growth factor in canine mammary tumors. Vet. Pathol., 39:488-493.

3. Wergin, M.C. and Kaser-Hotz. (2004) Plasma vascular endothelial growth factor (VEGF) measured in 70 dogs with spontaneously occurring tumors. In Vivo, 18:15-20.

4. Tamburini, B.A,, Trapp, S., Phang, T.L., Schappa, J.T., Hunter, L.E. and Modiano, J.F. (2009) Gene expression profiles of sporadic canine hemangiosarcoma are uniquely associated with breed. PLoS One, 4(5):e5549.

5. Yonemaru, K., Sakai, H., Yanai, T. and Masegi, T. (2006) Expression of vascular endothelial growth factor, basic fibroblast growth factor, and their receptors (FLT-1, FLK-1, and FLG-1) in canine vascular tumors. Vet. Pathol., 43: 971980.

6. Koch, S. and Claesson-Welsh, L. (2012) Signal transduction by vascular endothelial growth factor receptors. Cold Sprinng Harb Perspect Med., 2:a006502.

7. Kerber, M., Reiss, Y., Wickersheim, A., Jugold, M., Kiessling, F., Heil, M., Tchaikovski, V., Walternberger, J., Shibuya, M., Plate, K.H. and Machein, M.R. (2008). Flt-1 signaling in macrophages promotes glioma growth in vivo. CancerRes., 68:7342-7351.

8. Murakami, M., Iwai, S., Hiratsuka, S., Yamauchi, M., Nakamura, K., Iwakura, Y. and Shibuya, M. (2006) Signaling of vascular endothelial growth factor receptor-1 tyrosine kinase promotes rheumatoid arthritis through activation of monocytess/macrophages. Blood, 108:(18491856).

9. Hurwitz, H., Fehrenbacher, L., Novotny, W., Cartwright, T., Hainsworth, J., Heim, W., Berlin, J., Baron, A., Griffing, S., Holmgren, E., Ferrara, N., Fyfe, G., Rogers, B., Ross, R. and Kabbinavar, F. (2004) Bevacizumab plus irinotecan, fluorouracil, and leucovorin for metastatic colorectal cancer. N. Eng. J. Med., 350:2335-2342.

10. Miller, K., Wang, M., Gralow, J., Dickler, M., Cobleigh, M., Perez, E.A., Shenkier, T., Cella, D. and Davidson, N.E. (2007) Paclitaxel plus bevacizumab versus paclitaxel alone for metastatic breast cancer. N. Engl. J. Med., 357:26662676.

11. Jain, R.K., Duda, D.G., Clark, J.W. and Loeffler, J.S. (2006) Lessons from phase III clinical trials on anti-VEGF therapy for cancer. Nat. Clin. Pract. Oncol., 3:24-40.

12. Oh, S., Stish, B.J., Sachdev, D., Chen, H., Dudek, A.Z. and Vallera, D.A. (2009) A novel reduced immunogenicity bispecific ligand targeted toxin simultaneously recognizing human epidermal growth factor and interleukin-4 receptors in a mouse model of metastatic breast carcinoma. Clin. Cancer Res., 15(19): 6137-6147.

13. Spangler, C.W., Starkey, J.R., Rebane, A., Drobizhev, M., Meng, F. and Gong, A. (2008) Synthesis, characterization and two-photon PDT efficacy studies of triads incorporating tumor targeting and imaging components. Proc. Soc. Photo. Opt. Instrum., Optical Methods for Tumor Treatment and Detection: Mechanisms and Techniques in Photodynamic Therapy XVII, 68450S; doi:10.1117/12.763472.

14. Hamada, Y., Gonda, K., Takeda, M., Sato, A., Watanabe, M., Yambe, T., Satomi, S. and Ohuchi, N. (2011) In vivo imaging of the molecular distribution of the VEGF receptor during angiogenesis in a mouse model of ischemia. Blood 118(13). 
15. Ritt, M.G., Wojcieszyn, J., Smith, R., Mayor, J., Barton, C.L. and Modiano, J.F. (2000) Sustained nuclear localization of p21/Waf-1 upon growth arrest induced by contact inhibition. Cancer Lett., 158, 73-84.

16. Fosmire, S.P., Dickerson, E.B., Scott, A.M., Bianco, S.R., Pettengill, M.J., Meylemans, H., Padilla, M., Frazer-Abel, A.A., Akhtar, N., Getzy, D.M., Wojcieszyn, J., Breen, M., Helfand, S.C. and Modiano, J.F. (2004) Canine malignant hemangiosarcoma as a model of primitive angiogenic endothelium. Lab. Invest., 84(5): 562-72.

17. Kasza, L. (1964) Establishment and characterization of canine thyroid adenocarcinoma and canine melanoma cell lines. Am. J. Vet. Res., 25:1178-85.

18. Scott, M.C., Sarver, A.L., Gavin, K.J., Thayanithy, V., Getzy, D.M., Newman, R.A., Cutter, G.R., Lindblad-Toh, K., Kisseberth, W.C., Hunter, L.E., Subramanian, S., Breen, M. and Modiano, J.F. (2011) Molecular subtypes of osteosarcoma identified by reducing tumor heterogeneity through an interspecies comparative approach. Bone, 49, 356-367.

19. Livak, K.J. and Schmittgen, T.D. (2001) Analysis of relative gene expression data using real-time quantitative PCR and the 2(-Delta Delta C(T)) Method. Methods, 25, 402-408.

20. Schappa, J.T., Frantz, A.M., Dickerson, E.B., Vallera, D.A. and Modiano, J.F. (2013) Hemangiosarcoma and its cancer stem cell sub-population are effectively killed by a toxin targeted through epidermal growth factor and urokinase receptors. Int. J. Cancer, 133, 1936-44.

21. Pastan, I., Chaudhary, V. and FitzGerald, D.J. (1992) Recombinant toxins as novel therapeutic agents. Annu. Rev. Biochem., 61:331-54.

22. Waldron, N., Seunguk, Oh. and Vallera, D. (2012) Bispecific targeting of EGFR and UPAR in a mouse model of head and neck squamous cell carcinoma. Oral Oncol., 48 (12):1202-1207.

23. Troy, G.C., Huckle, W.R., Rossmeisl, J.H., Panciera, D., Lanz, O., Robertson, J.L. and Ward, D.L. (2006) Endostatin and Vascular Endothelial Growth Factor Concentrations in Healthy Dogs, Dogs with Selected Neoplasia, and Dogs with Nonneoplastic Diseases. J. Vet. Intern. Med., 20:144-150.

24. Clifford, C.A., Hughes, D., Beal, M.W., Mackin, A.J., Henry, C.J., Shofer, F.S. and Sorenmo, K.U. (2001) Plasma vascular endothelial growth factor concentrations in healthy dogs and dogs with hemangiosarcoma; J. Vet. Intern. Med., 15(2):1315.

25. Paoloni, M. and Khanna, C. (2008) Translation of new cancer treatments from pet dogs to humans. Nature Reviews, $8: 147-156$.

$* * * * * * * *$ 